\title{
Tool Development for Human Audible Spectrum Compensation
}

\author{
Francisco Gonçalves, Filomena \\ Soares \\ Algoritmi R\&D Centre \\ University of Minho \\ Guimarães, Portugal \\ fsoares@dei.uminho.pt
}

\author{
Vitor Carvalho \\ Algoritmi R\&D Centre \& *EST \\ University of Minho \& *IPCA \\ Guimarães, *Barcelos, Portugal \\ vcarvalho@ipca.pt
}

\author{
José Machado \\ MEtRICs R\&D \\ University of Minho \\ Guimarães, Portugal \\ jmachado@dem.uminho.pt
}

\begin{abstract}
Communication relies on good understanding. Humans relate to each other through visual, audible and tactile communication. It is imperative that the audible communication message reaches the receiver in good conditions, in order to keep a healthy, smooth and understandable speech. There are some disturbances in human speech and communication when hearing damage is present. Nowadays, hearing loss is a frequent injury, caused by noise pollution, daily stress or noisy workplaces. Yet, it can be treated by several ways. This project consists in developing a tool that captures the emitter's voice audible spectrum, filters the noise and other frequencies, and compensates the message, enabling the listener/receiver understanding. The purpose of this research is not aimed to substitute nor compete with hearing aids in the market, which are well-developed, certified and prescribed by Otorhinolaryngology clinicians. The focus of this study is to identify the issues of human hearing loss and to develop an algorithm for hearing compensation by using filtering techniques in a simulated environment applied to a hearing model.
\end{abstract}

Keywords - digital signal processing; hearing aid; noise filtering; speech; human ear.

\section{INTRODUCTION}

One of the big issues in human understanding is the ability to realize the sounds so that it is possible to translate the message to the brain [1]. It is very important for humans to relate to each other and feel comfortable when communicating. Hearing is one of the success's key.

The ability to hear is critical to understand the world around us as well as interacting with each other. Hearing impairment is the most frequent sensory deficit in human populations and affects newborns, children, adults and elderly [2], [3].

However, hearing can be compromised by issues that may lead to an inefficient reception of the message. One cause is the hearing loss. There are many causes for hearing loss. Sometimes it is just a case of a relatively simple procedure like cleaning the audible canal in the ear, where it is wax accumulation that should be removed. There is also the possibility for an accident or even the use of antibiotics. On the other hand, when the nerves are affected there is no way to recover. Hearing loss is one of the causes of social and speech/understanding problems.

The research objective of this paper aims to be a first step in order to apply digital signal processing (DSP) and filtering techniques to a hearing model in order to enhance speech [4].

The paper is organized as follows: Section II describes the hearing loss concept; Section III presents the spectrum of frequencies of hearing; Section IV shows examples of hearing aids as well as some mobile applications and finally, Section $\mathrm{V}$ presents the final remarks.

\section{HEARING LOSS}

When describing hearing loss, we generally look at three aspects: type of hearing loss, degree of hearing loss, and configuration of hearing loss.

There are three basic types of hearing loss: conductive, sensorineural, and mixed. Conductive hearing loss occurs when the sound is not sent easily through the outer ear canal to the eardrum and the tiny bones (ossicles) of the middle ear. Conductive hearing loss makes sounds softer and less easy to hear. This type of hearing loss can often be corrected by medically or surgically interventions. Some possible causes of conductive hearing loss are [2], [3]:

- Fluid in the middle ear from colds or allergies;

- Ear infection (otitis media);

- Malfunction in the eustachian tube;

- Hole in the eardrum;

- Too much earwax;

- External otitis;

- Foreign body in the ear canal (insects, among others);

- Malformation of the outer ear, ear canal, or middle ear.

Sensorineural hearing loss (SNHL) happens when there is damage to the inner ear (cochlea) or to the nerve pathways from the inner ear to the brain. Most of the time, SNHL cannot be medically or surgically corrected. This is the most common 
type of permanent hearing loss. SNHL reduces the ability to hear faint sounds. Some possible causes of SNHL are:

- Drugs that are toxic to hearing;

- Hearing loss that runs in the family (genetic or hereditary);

- Aging;

- Head trauma;

- Malformation of the inner ear;

- Exposure to loud noise.

Mixed hearing loss occurs when a conductive hearing loss happens in combination with an SNHL. In other words, there may be damage in the outer or middle ear and in the inner ear (cochlea) or auditory nerve [5].

Patients with SNHL, however, also may experience tinnitus, dizziness, or even true vertigo. Careful questioning may reveal that the patient has a specific difficulty understanding speech. This may be apparent only in certain situations, such as when speaking on the phone or in a conversation in a noisy environment or with certain individuals (children or women whose voices tend to be more silent and high pitched).

Hearing loss is an important public health concern with substantial economic and social costs. In children, hearing impairment delays language development and educational progress. In adults, it causes difficulties in professional and social life as well as self-esteem. Apart from consequences to the individual person, hearing loss also leads to high costs to society.

Hearing impairment can be caused by a number of factors including infections during childhood such as measles, mumps and meningitis, chronic otitis media, exposure to excessive or prolonged noise, head/neck injuries, use of medications such as certain types of chemotherapies and antibiotics, industrial solvents, congenital abnormalities and infections and perinatal problems, certain nutritional deficiencies, genetic disorders and aging. Use of hearing devices [6] such as aids and cochlear implants as well as sign language, lip reading and special amplification systems in schools are strategies to help affected people manage their communication [7].

\section{SPECTRUM OF FREQUENCIES}

Hearing is the ability to perceive sounds. Sound occurs over a wide spectrum of frequencies. The human ear is sensitive to a frequency band within that spectrum expressed in decibels $(\mathrm{dB})$. Humans are capable of hearing sounds from a range of frequencies. The range is typically considered to be between $20 \mathrm{~Hz}$ and $20000 \mathrm{~Hz}$. Frequencies higher than $20 \mathrm{kHz}$ are considered as ultrasonic, while frequencies below audio are referred to as infrasonic. Loss of the ability to hear sound frequencies in the normal range of hearing is called hearing impairment.

There is a diversity of definitions of hearing impairment, thus, comparison among studies is difficult and may be invalid. The definition used by any study should always be checked before attempting to make such comparisons. The World Health Organization (WHO) defines disabling hearing impairment in adults as a permanent unaided hearing threshold level (average for frequencies $500 \mathrm{~Hz}, 1000 \mathrm{~Hz}, 2000 \mathrm{~Hz}$, $4000 \mathrm{~Hz}$ ) for the better ear of $41 \mathrm{~dB}$ or greater (WHO, 2001). In children under 15 years of age, disabling hearing impairment is defined as permanent unaided hearing threshold level (average for frequencies $500 \mathrm{~Hz}, 1000 \mathrm{~Hz}, 2000 \mathrm{~Hz}$, $4000 \mathrm{~Hz}$ ) for the better ear of $31 \mathrm{~dB}$ or greater [5]. Figure 1 shows the frequencies and threshold levels of hearing [8].

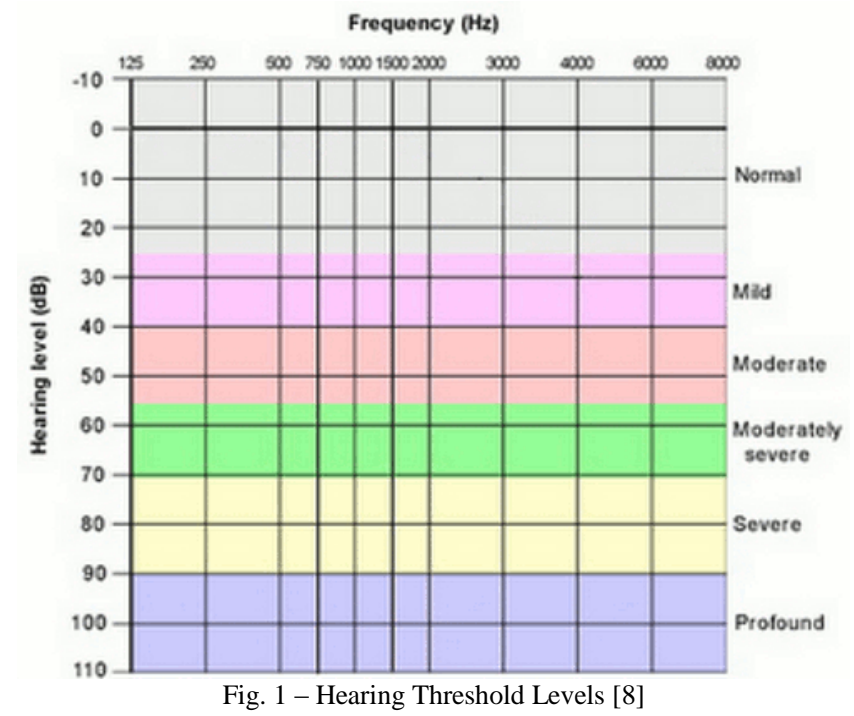

Figure 2 shows a typical audiometric examination of a patient with mild to moderate hearing loss.

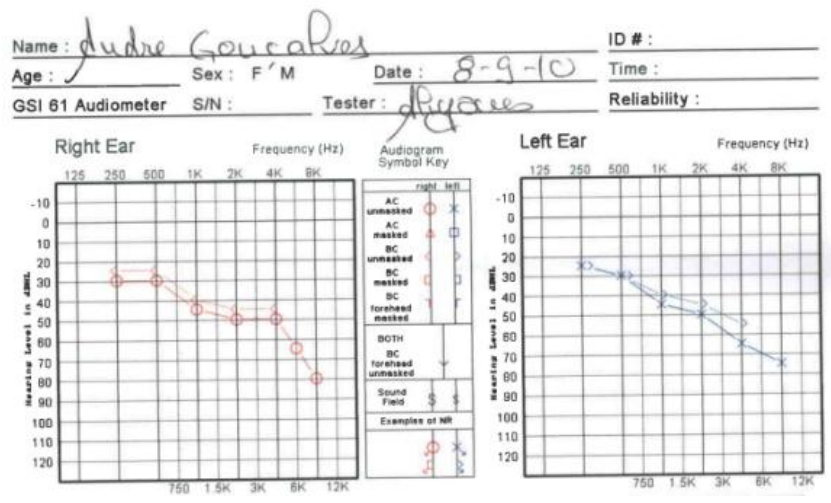

Fig. 2 - Audiometric Examination Test (example)

In this example, there is no difference between the air conduction and the bone conduction thresholds; it indicates that the hearing loss is due to a problem in the cochlea. This is the most common type of hearing loss in adults and it is known as a sensorineural hearing loss [9]. 


\section{HEARING AIDS}

Hearing aids can be applied to overcome this impairment. Because hearing aids today are digital and programmable, the amplification can be fine-tuned and tailored for each wearer. Basic hearing aids usually require some manual adjustments depending on the type and degree of loss experienced by a person. Basic hearing aids allow for some customization but are limited in the amount of adjustments to fit unusual patterns of hearing loss. Advanced hearing aids offer several different levels of technology. As the level of technology increases, hearing devices are more automatic, equipped with more features and allow for a more-personal experience.

Some of the greatest advantages of digital signal processing in hearing aids applications are [10]:

- Gain Processing (the potential for increased audibility of sounds of interest without discomfort);

- Digital Feedback Reduction (monitor for feedback while the listener is wearing the hearing aid);

- Digital Noise Reduction (intended to reduce gain in the low frequencies or bandwidth when noise is detected);

- Digital Speech Enhancement (a relatively new to hearing aid, it is intended to improve temporal or spectral content of speech in order to enhance speech);

- Directional Microphones and DSP (ability of directional hearing aids to improve the effective signal-to-noise ratio provided to the listener);

- Digital Hearing Aids as Signal Generators.

The hearing aids produced today utilize wireless technology. This allows two hearing aids to operate together as one complete system, instead of acting as two independent devices. Additionally, wireless technology gives hearing aid users a greater ability to customize their experience and program their device.

Wireless hearing aids are often capable of wirelessly communicating with external devices as well, as Bluetooth technology. Bluetooth enables hearing aid wearers to connect personal electronic devices such as mobile phones and stream signals directly to the hearing aid. Wireless hearing aids can use compatible assistive listening devices, often called streamers, to provide a communication link between the wireless technology in the hearing aids and any Bluetoothenabled device.

\section{A. Types of Hearing Aids}

While hearing aids can be split into two broad categories in-the-ear styles (ITE) and behind-the-ear styles (BTE) - there are several types available worn in different locations in or behind the ear, allowing the wearer to find the most comfortable and beneficial fit.
The following hearing aid types are considered in-the-ear styles and vary from completely invisible and in the canal to on the outer edge of the ear bowl[11][12]:

- $\quad \boldsymbol{B T E}$ - Behind the Ear. Placed behind the ear. Very often used in children where safety is concerned (Fig. $3)$.

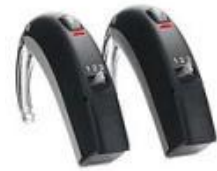

Fig. 3 - BTE - Behind the Ear

- Behind-The-Ear Aid (Open Fitting). Very similar to the BTE but with small and transparent tube for cosmetic reasons (Fig. 4).

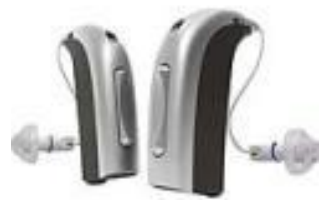

Fig. 4 - Behind The Ear (open fitting)

- $\quad$ ITC - In The Canal. Device partially inside the canal (Fig. 5).

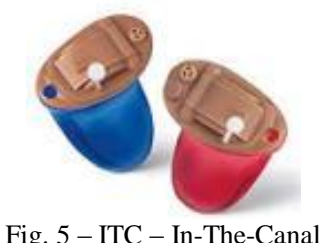

- $\quad \boldsymbol{C I C}$ - Completely in the canal. Almost as ITC, this type is enclosure in a small box and completely inside the canal. They offer the best solutions for cosmetic purposes (Fig. 5).

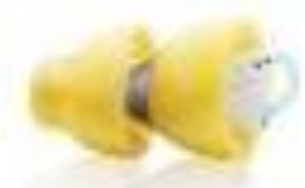

Fig. 5 - CIC - Completely In the Canal

- $\quad \boldsymbol{I T E}$ - In The Ear. All parts are outsider the canal. This type is usually used by those how are not comfortable with CIC models (Fig. 6).

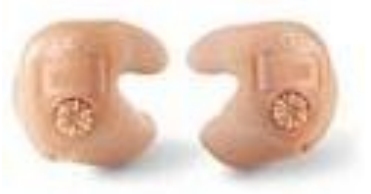

Fig. 6 - ITE - In-The-Ear 


\section{B. Hearing Aids in Mobile Applications}

There are also hearing aids available as mobile applications where it is possible to control some parameters via Bluetooth [13], [14] (Fig. 7-8).

These mobile applications are used to access to a vast array of parameters controlling, via Bluetooth. It is possible to control volume, spatial customized configurations, among others. They are free of charge and intended for use with Siemens hearing aid products (Fig. 7) or GN ReSound (Fig. $8)$.
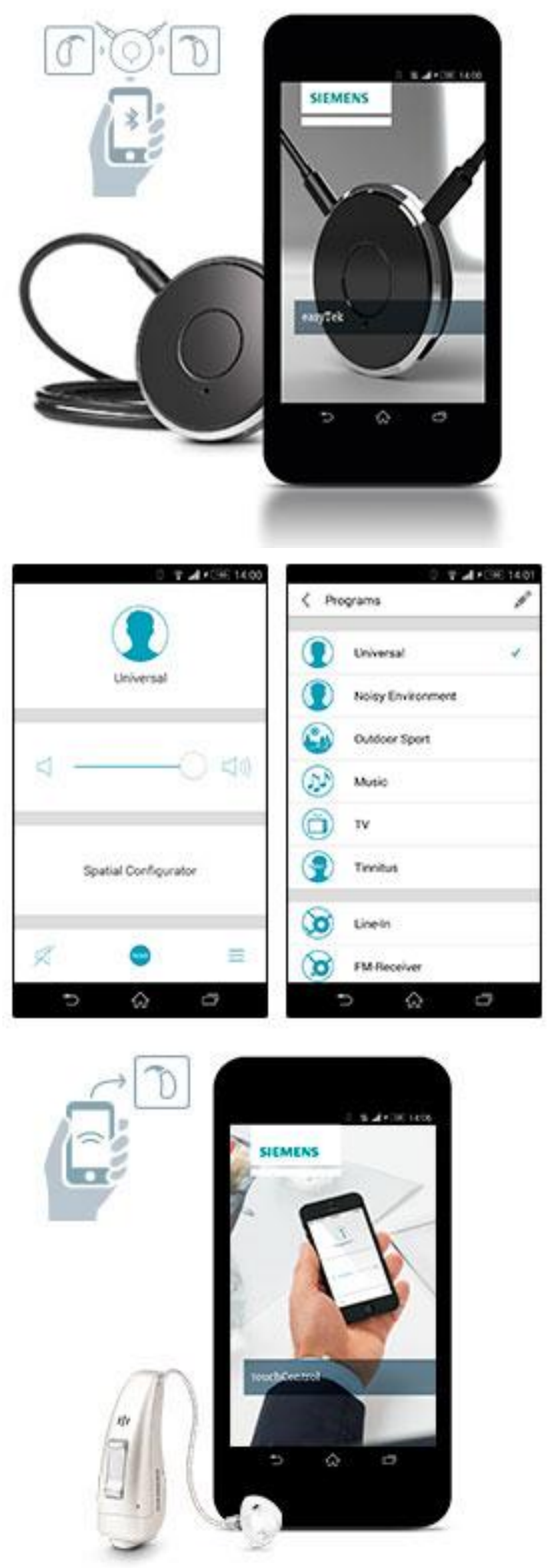

Fig. 7 - TouchControl App e easyTEK App from Siemens 


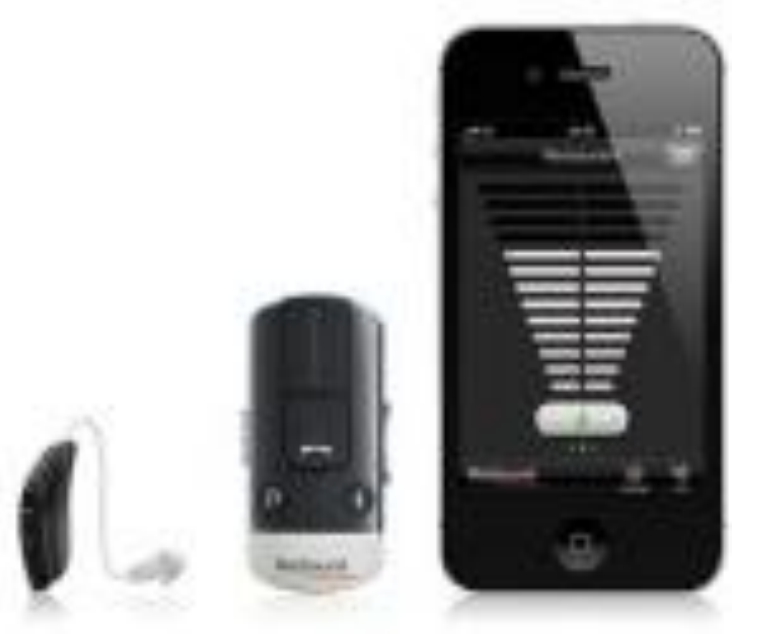

Fig. 8 - ReSound Control Mobile App from GN ReSound, available for iOS.

There are some tools, in the form of application for mobile devices, usually limited, with some tests available so that the user have an idea of his hearing situation. Some applications (almost all of them are payware) can be used as a hearing aid as mobile's headphones and microphone (Fig. 9 to Fig. 11, courtesy of BioAidProject, Unitron Limited and Ginger Labs, respectively).

This application is intended to give an audiometric test so that the user may have an approach of his/her hearing loss and thus, if necessary, contact the Otorhinolaryngology clinicians to evaluate the problem.

Through a simple interface, the user can adjust a few parameters to enchance hearing.

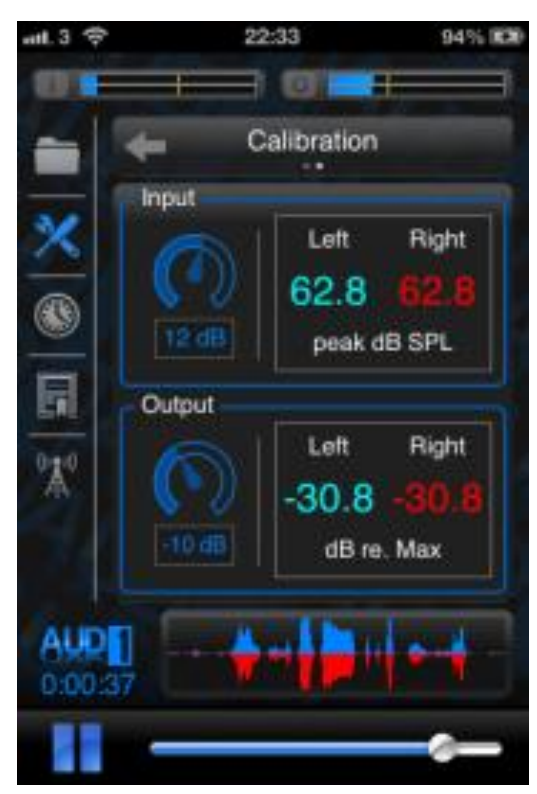

Fig. 9 - Screenshot from AUD1 [15], from BioAid Project, available for iOS devices.

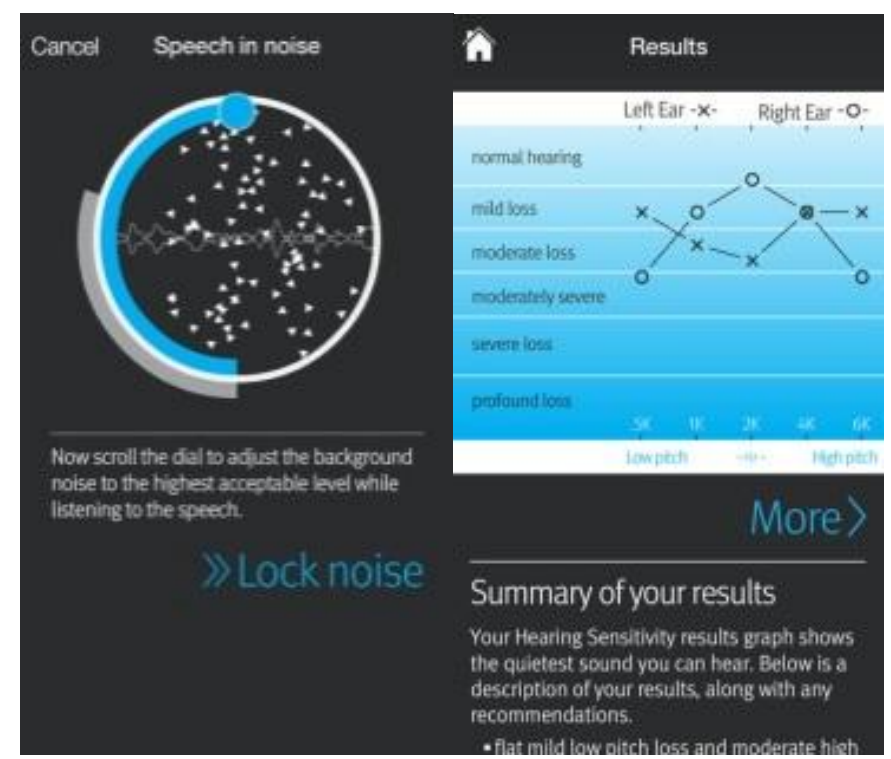

Fig. 10 - uHear [16] from Unitron Hearing Limited

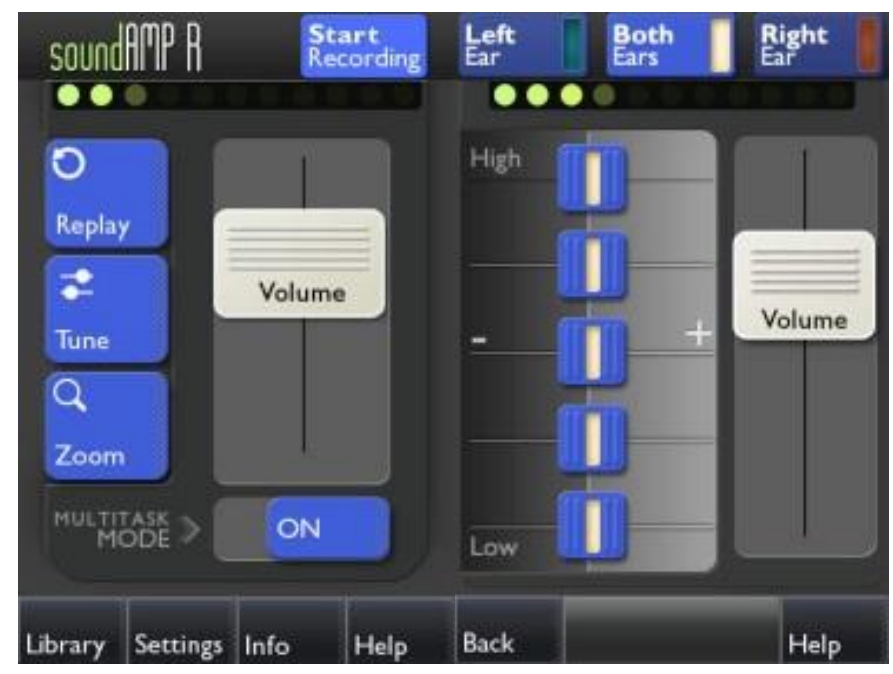

Fig. 11 - soundAMP R [17] from Ginger Labs

\section{FINAL REMARKS}

The research objective is to apply DSP and filtering techniques to a hearing model in order to enhance speech. Some techniques should be used like noise cancelation, amplification, compression, frequency transportation [18], [19].

Hearing aids are based on complex algorithms and well defined structures. Algorithms intended to simulate the human auditory system translating behaviors. Hearing aids cannot overcome the perceptual distortions caused by hearing loss but enables the user to understand the speech in a better way [20$23]$. 


\section{Acknowledgment}

This work has been supported by FCT - Fundação para a Ciência e Tecnologia in the scope of the project: PEstUID/CEC/00319/2013.

\section{References}

[1] Heinz G. Gockler, Henning Puder, Hugo Fastl, Sven Erik Nordholm, Torsten Dau, Walter Kellermann, Editorial Digital Signal Processing for Hearing Instruments, Hindawi Publishing Corporation, EURASIP Journal on Advances in Signal Processing Volume 2009, Article ID 898576, 3 pages doi: $10.1155 / 2009 / 898576$.

[2] Conserve Energy Green, Understanding Noise Pollution, http://www.conserve-energy-future.com/causes-and-effects-of-noisepollution.php (access in February 2015).

[3] Guidelines For Community Noise, Edited by Birgitta Berglund, Thomas Lindvall, Dietrich Schwela, World Health Organization, Geneva Cluster of Sustainable Development and Healthy Environment (SDE), Department of the Protection of the Human Environment (PHE), Occupational and Environmental Health (OEH), http://www.who.int/docstore/peh/noise/Comnoise-1.pdf (access in February 2015).

[4] Ajay Natarajan, John Hansen, Kathryn Arehart, Jessica Rossi-Katz, An Auditory-Masking-Threshold-Based Noise Suppression Algorithm GMMSE-AMT[ERB] for Listeners with Sensorineural Hearing Loss, EURASIP Journal on Applied Signal Processing, 2005 Hindawi Publishing, 2005:18, 2938-2953.

[5]https://store.acponline.org/ebizatpro/images/productimages/books/sample\% 20chapters/Oto_Ch03.pdf (access in February 2015).

[6] Hearing loss - Treatment, http://www.nhs.uk/Conditions/Hearingimpairment/Pages/Treatment.aspx (access in February 2015).

[7]http://www.who.int/medicines/areas/priority_medicines/BP6_21Hearing.pd $\mathrm{f}$ (access in February 2015).

[8]http://auditoryneuroscience.com/acoustics/clinical_audiograms (access in February 2015).

[9]http://www.chimehealth.co.uk/web/data/audiogram-hearing-loss-examples2.pdf (access in February 2015).

[10]http://www.asha.org/public/hearing/Digital-Hearing-Aids-CurrentStateof-the-Art/ (access in February 2015).

[11]http://www.healthyhearing.com/help/hearing-aids (access in February 2015).

[12]https://us.hearing.siemens.com/hearing-products/hearing-aidaccessories/touchcontrol-app/, (access in February 2015).

[13]https://us.hearing.siemens.com/hearing-products/hearing-aidaccessories/easytek-app/ (access in February 2015).

[14]http://www.gnresound.com/hearing-aids/accessories/unite-phone-clipplus/resound-control-app (access in February 2015).

[15]http://www.aud1.com/, http://bioaid.org.uk/ (access in February 2015).

[16]https://itunes.apple.com/pt/app/id30981182?mt=8 (access in February 2015).

[17]https://itunes.apple.com/pt/app/id318126109?mt=8 (access in February 2015).

[18]http://www.ijera.com/papers/Vol3_issue2/GR3212241229.pdf (access in February 2015).

[19] http://cdn.intechopen.com/pdfs-wm/15952.pdf (access in February 2015).

[20]http://audioplastic.org/blog/2013/01/29/bioaid-algo-2/ (access in February 2015).

[21]http://www.math.uci.edu/ jxin/iccm04.pdf (access in February 2015).

[22]http://www.math.utah.edu/ borisyuk/PAPERS/LectureNotes_preprint.pdf (access in February 2015).

[23]http://www.gizmag.com/hearing-aid-noise-filtering-

algorithm/29799/(access in February 2015). 